\title{
Band gap behaviour of optimal one-dimensional composite structures with an additive manufactured stiffener
}

\author{
T. Ampatzidis*a , R. K. Leach ${ }^{\mathrm{b}}$, C. J. Tuck ${ }^{\mathrm{c}}$, D. Chronopoulos ${ }^{\mathrm{a}}$ \\ ${ }^{a}$ Institute for Aerospace Technology \& The Composites Group, University of Nottingham, \\ $N G 81 B B, U K$ \\ ${ }^{b}$ Manufacturing Metrology Team, Faculty of Engineering, University of Nottingham, \\ Nottingham NG8 $1 B B, U K$ \\ ${ }^{c}$ Centre for Additive Manufacturing, Faculty of Engineering, University of Nottingham, \\ Nottingham NG8 1BB, UK
}

\begin{abstract}
In this work, the banded behaviour of composite one-dimensional structures with an additive manufactured stiffener is examined. A finite element method is used to calculate the stiffness, mass and damping matrices, and periodic structure theory is used to obtain the wave propagation of one-dimensional structures. A multi-disciplinary design optimisation scheme is developed to achieve optimal banded behaviour and structural characteristics of the structures under investigation. Having acquired the optimal solution of the case study, a representative specimen is manufactured using a carbon fibre cured plate and additive manufactured nylon-based material structure. Experimental measurements of the dynamic performance of the hybrid composite structure are conducted using a laser vibrometer and electrodynamic shaker setup to validate the finite element model.
\end{abstract}

Keywords: Composite Structures, Band Gap Behaviour, Additive Manufacturing, Hybrid Structures, Wave Propagation

Email address: Theofanis.Ampatzidis@nottingham.ac.uk (T. Ampatzidis*) 


\begin{tabular}{|c|c|c|}
\hline \multicolumn{3}{|c|}{ Nomenclature } \\
\hline$\beta_{i}$ & design parameters & propagation constants in the \\
\hline f & force vector & $x$ direction \\
\hline q & physical displacement vector & $a_{i}, b_{i}, c_{i}, d_{i}$ design cost coefficients \\
\hline $\mathbf{R}$ & transformation matrix & $b_{-} g \quad$ band gap \\
\hline$\eta$ & loss factor & $\begin{array}{cl}b_{-} g_{m_{-} f} \text { band gap mid-point fre- } \\
\text { quency }\end{array}$ \\
\hline$\lambda_{i}$ & $\begin{array}{l}\text { eigenvalue corresponding to } \\
\text { frequency } f_{i}\end{array}$ & stiffener's bending stiffness \\
\hline I & identity matrix & $E, E_{x}, E_{y}, E_{z}$ Young's moduli \\
\hline $\mathbf{M}, \mathbf{K}$ & $\begin{array}{l}\text { and } \mathbf{C} \text { mass, stiffness and } \\
\text { damping matrices of the unit }\end{array}$ & $\begin{array}{l}f_{i} \quad \text { frequency } \\
G_{x y} G_{x z} G_{y z} \text { shear moduli }\end{array}$ \\
\hline & cell & $k_{x} \quad$ wavenumbers in the $x$ direc- \\
\hline & damping matrices of the in- & length of the unit cell \\
\hline & dividual finite elements & length of the top of the stiff- \\
\hline $\mathbf{p}$ & design parameter vector & ener \\
\hline $\mathrm{x}$ & right eigenvector & mass \\
\hline $\mathcal{F}(\mathbf{p})$ & objective cost function & time \\
\hline$\omega$ & angular frequency & stiffener's thickness \\
\hline$\rho$ & mass density & $v_{x y} v_{x z} v_{y z}$ Poisson's ratios \\
\hline
\end{tabular}

\section{Introduction}

Noise and vibration transmission within payload and passenger compartments is a major issue for modern transport vehicles. To ensure the quality of their products, manufacturers in the transport industry are simultaneously 
5 trying to optimise the mechanical and the vibroacoustic performance of structural assemblies. It has been demonstrated that judiciously designed periodic structures can induce vibration attenuation and stop-band behaviour in specific frequency ranges (so-called band gaps or stop bands).

Floquet [1] was the first to publish on periodic structures, in which the one-dimensional (1D) Mathieu's equations were studied to predict band gap behaviour. Floquets work was followed by that of Rayleigh [2], who developed a similar form to Floquet's theorem. During the twentieth century, Mead [3, 4], Mace et al. [5] and Langley and Cotoni et al. [6, 7] produced mathematical tools based on Brillouin's periodic structure theory (PST) [8]. Using these methods, researchers have the ability to predict the vibroacoustic and dynamic performance of several applications in relatively short times. Application examples are presented with composite panels and shells $[9,10]$, structures with pressurised shells [11], and complex periodic structures $[12,13,14,15]$. Hussein et al. [16] produced an extensive review of developments in band gap technology.

There are two major mechanisms that have been identified to generate band gap behaviour in periodic structures: Bragg scattering and local resonance. Bragg scattering is observed when a structure exhibits periodic impedance mismatches and the waves are scattered at the borders of the unit-cell (the part of the structure that is periodically repeated). This scattering can be caused by means of inclusions, and geometrical or material inconsistencies, and leads to the interaction of the reflected waves with the incoming waves. When specific circumstances are met, this interaction causes the partial or complete annihilation of wave propagation $[16,17]$. It can easily be shown that the frequency at which the band gap is observed depends on the length and the material/geometrical mismatch of the unit cell of the periodic structure. This leads to the need for prohibitively large dimensions to achieve low frequency band gaps. Therefore, researchers' focus was shifted to local resonance [18], where a solid core material with relatively high density is usually preferred, suppressed by an elastically soft material. When this sub-wavelength inclusion/addition resonates, it exhibits behaviour that cancels the propagation of waves, giving rise to effective 
negative elastic constants or group velocities at certain frequency ranges which are significantly lower than those observed in Bragg scattering. Liu et al. [19] examined the transition between the two band gap production mechanisms and there has been research on coupling of the two mechanisms [20,21]. In this work 40 an optimisation method is developed capable of examining both band gap production mechanisms, and the Bragg scattering mechanism is observed in case study geometry to demonstrate its application.

Structures that exhibit band gap behaviour tend to be of significantly complex geometry and cannot be realised using conventional manufacturing techniques. Therefore, additive manufacturing (AM) technologies attract an increasing number of researchers [22]. AM eliminates several design limitations and is currently in extensive use in research and industry for small-scale production with a wide variety of manufacturing operations. AM researchers take advantage of the freedom in design that can be attained with relatively low cost, by avoiding the manufacturing cost of additional tools. Matlack et al. [21] examined the broadband vibration absorption of AM meta-structures exhibiting local resonance, while Qureshi et al. [23] modelled and manufactured a cantilever-in-mass metamaterial to achieve wave attenuation. Claeys et al. [24] examined, both numerically and experimentally, several versions of a vibroacoustic metamaterial with local resonance and compared the two sets of results, while Warmuth et al. [25] used AM methods to manufacture a 3D single phase phononic band gap material with cellular design exhibiting a wide stop band at high frequencies. Bilal et al. [26] experimentally observed the trampoline phenomenon on the band gap behaviour of AM metamaterial plates. The wide variety of designs that can be produced using AM methods has led to the opportunity for researchers to examine 3D band gaps in complex structures [27, 28]. This wide use of AM has led researchers to examine the effect of the property variability of $\mathrm{AM}$ on the experimental results [29, 30], where a better agreement was obtained between analysis and experimental results by considering uncertainties in the resonators and the host structure.

Efficient and accurate optimisation schemes are essential when determining 
design solutions and researchers use several methods. The optimal design of an Euler-Bernoulli simple band gap beam, made of linearly elastic material, has been recently examined [31], where the author used a bound optimisation method which optimised the gap between natural frequencies. Hussein et al. $[32,33,34]$ optimised the band gap behaviour of periodic layered structures, where methods to achieve band gap behaviour within specific frequencies were developed. More specifically, Hussein et al. employed a multi-objective genetic algorithm that generates a population of possible solutions and searches for the optimum one. This method was tailored so that several objectives were examined, such as the percentage of the band gaps in specific frequencies, low frequency band gaps and control of the speed of energy propagation in the structure. Jensen and Sigmund [35] optimised the band gap behaviour of phononic structures using topology methods. Langley et al. [7] used a quasi-Newton algorithm with 30 random-start runs to obtain the optimal vibration absorption of the structure, while Wormser et al. [27] optimised the phononic band gap behaviour of cellular structure using gradient based methods. To the authors' knowledge, all the optimisation methods for phononic band gap behaviour focus solely on the band gap tailoring itself.

The novelty of the work presented in this paper is:

- An optimal design of a band gap structure is obtained, so that it serves both as a stiffener and band gap production mechanism, constituting a structural part.

- The optimal design of the structure is obtained by applying a developed computationally efficient unit cell based optimisation scheme.

- The developed multi-disciplinary design optimisation scheme uses scalarisation for simultaneous mass and vibration minimisation and static stiffness maximisation. Several starting points are used and parametric analysis is completed to evaluate the optimal solution.

95 More specifically, the multi-disciplinary optimisation of vibration attenuation 
through band gap and static structural performance of a 1D composite structure with powder bed fusion of polyamide 12 (PA12) material additions is examined. The structure is modelled using finite element (FE) method and PST is used for predicting its wave propagation characteristics. A constrained non-linear optimisation method is used for the optimal geometric characteristics to be acquired. The optimal solution is realised using a powder bed fusion (laser sintering) method and is tested using a laser vibrometer and electrodynamic shaker setup to validate the FE model.

The paper is organised as follows: in Section 2, the FE and optimisation method are presented. In Section 3, the examined case is presented, along with the results of the analysis. In Section 4, the FE analysis of the case study and the experimental methodology is described and the results are discussed. Finally, in Section 5, the conclusions of the work are drawn.

\section{Methodology}

\subsection{Periodic structure theory}

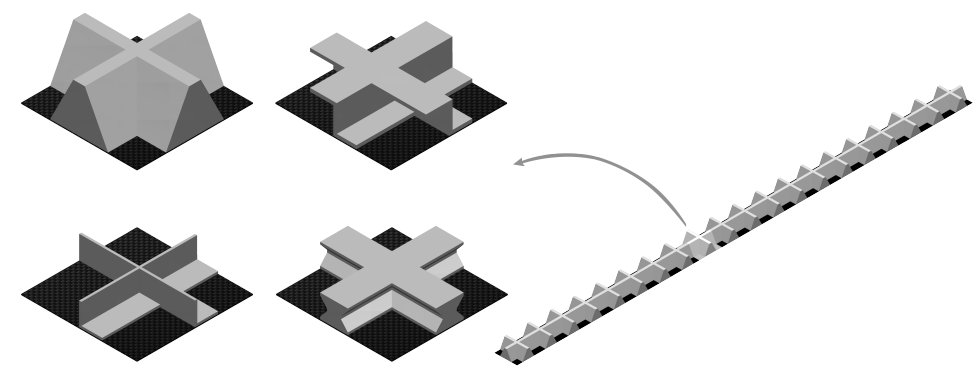

Figure 1: Several schemes of periodic structures.

The PST used in this work is presented in detail elsewhere [6]. A generic structure with 1D periodicity is considered. A unit cell is extracted from the structure (see Fig. 1) and its behaviour modelled with the FE method. A steady-state free harmonic vibration of frequency $\omega$ is considered in what follows 
and all response quantities are represented by complex amplitudes, so that

$$
\mathbf{q}(t)=\mathbf{q} \mathrm{e}^{\mathrm{i} \omega t}
$$

where $t$ is time and the degrees of freedom $\mathbf{q}$ can be partitioned into interior

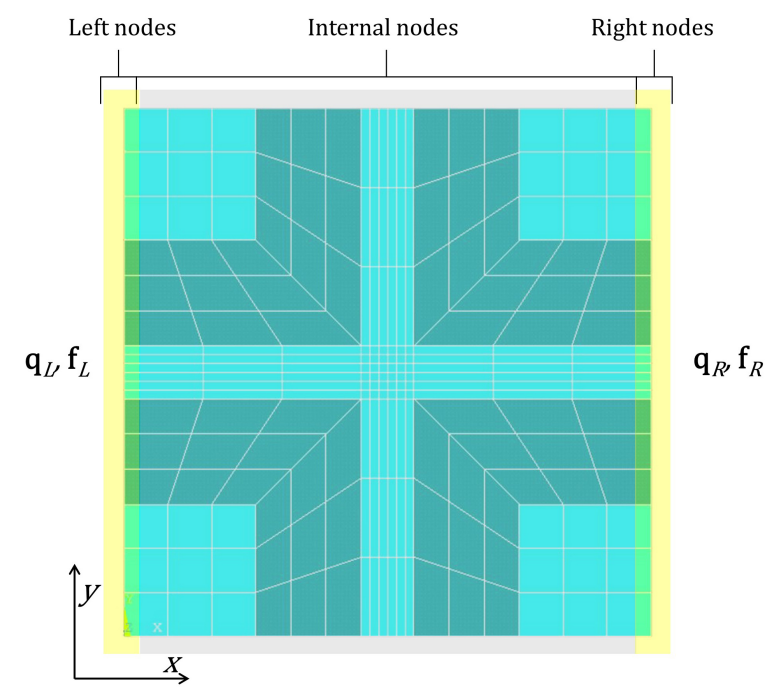

Figure 2: The employed FE model for investigation of 1D wave propagation.

(I), left (L) and right (R) (see Fig. 2). According to Floquet's theorem, the equation that relates the displacements on the sides of the modelled unit cell is the following [3]:

$$
\mathbf{q}_{R}=\mathrm{e}^{-\mathrm{i} \varepsilon_{x}} \mathbf{q}_{L},
$$

where the term $\varepsilon_{x}=k_{x} L_{x}$ is referred to as 'phase constant', $L_{x}$ is the periodic element's length in the $x$ direction and $k_{x}$ is the wavenumber. The complete vector of the local degrees of freedom can be ordered

$$
\mathbf{q}=\left[\begin{array}{lll}
\mathbf{q}_{I}^{T} & \mathbf{q}_{L}^{T} & \mathbf{q}_{R}^{T}
\end{array}\right]^{T} .
$$

The equation of motion for the cell including damping [36] is given by

$$
\left[\mathbf{K}+\mathrm{i} \omega \mathbf{C}-\omega^{2} \mathbf{M}\right] \mathbf{q}=\mathbf{f},
$$


where $\mathbf{K}, \mathbf{M}$ and $\mathbf{C}$ are the stiffness, mass and damping matrices respectively and $\mathbf{f}$ is the vector of the nodal forces. Eq. (4) can be used when proportional damping is available [37], where damping is expressed as a linear combination of the mass and stiffness matrices, that is,

$$
\mathbf{C}=\alpha_{1} \mathbf{M}+\alpha_{2} \mathbf{K}
$$

where $\alpha_{1}$ and $\alpha_{2}$ are real scalars. In the case of structural damping $[9,36]$, the equation of motion becomes

$$
\left[\mathbf{K}+\mathrm{i} \mathbf{C}-\omega^{2} \mathbf{M}\right] \mathbf{q}=\mathbf{f},
$$

and the structural damping matrix $\mathbf{C}$ is given by

$$
\mathbf{C}=\sum_{i=1}^{n_{e}} \mathbf{K}_{i} \eta_{i}
$$

where $n_{e}$ is the number of the FEs of the unit cell, $\mathbf{K}_{i}$ is the stiffness matrix of the $i_{t h}$ element and $\eta_{i}$ is the loss factor of the corresponding element. Eq. (6) can be written as

$$
\left[\mathbf{K}(1+\mathrm{i} \eta)-\omega^{2} \mathbf{M}\right] \mathbf{q}=\mathbf{f} .
$$

In this work, we do not focus on the accuracy of the model of damping, since the main aim of the wave propagation analysis is to examine the existence of the band gap. In order to write the propagation relation in Eq. (1) in matrix form, we consider transformation matrix $\mathbf{R}$, which is given in the following equation:

$$
\mathbf{q}=\left[\begin{array}{cc}
\mathbf{I} & \mathbf{0} \\
\mathbf{0} & \mathbf{I} \\
\mathbf{0} & \mathbf{I}^{-\mathrm{i} \varepsilon_{x}}
\end{array}\right] \mathbf{x}=\mathbf{R x}
$$

In this way, we get

$$
\mathbf{q}=\mathbf{R} \mathbf{q}^{\prime}
$$

where

$$
\mathbf{q}^{\prime}=\mathbf{x}=\left[\begin{array}{ll}
\mathbf{q}_{I} & \mathbf{q}_{L}
\end{array}\right]^{T}
$$


In the absence of external forces (free wave propagation), we have

$$
\mathbf{R}^{H} \mathbf{f}=\mathbf{0}
$$

where $\mathbf{R}^{H}$ denotes the complex conjugate (Hermitian) transpose of $\mathbf{R}$. The resulting homogenous equations in the reduced set of degrees of freedom are then given by

$$
\left[\mathbf{K}^{\prime}+\mathrm{i} \omega \mathbf{C}^{\prime}-\omega^{2} \mathbf{M}^{\prime}\right] \mathbf{q}=\mathbf{0}
$$

and

$$
\left[\mathbf{K}^{\prime}(1+i \eta)-\omega^{2} \mathbf{M}^{\prime}\right] \mathbf{q}^{\prime}=\mathbf{0}
$$

where

$$
\mathbf{K}^{\prime}=\mathbf{R}^{H}\left(\varepsilon_{x}\right) \mathbf{K R}\left(\varepsilon_{x}\right), \quad \mathbf{M}^{\prime}=\mathbf{R}^{H}\left(\varepsilon_{x}\right) \mathbf{M R}\left(\varepsilon_{x}\right), \quad \mathbf{C}^{\prime}=\mathbf{R}^{H}\left(\varepsilon_{x}\right) \mathbf{C R}\left(\varepsilon_{x}\right)
$$

When a set of phase constants $\varepsilon_{x}$ is specified, we get a quadratic eigenvalue problem for Eq. (13), the solution method of which is described elsewhere [38]. Eq. (14) gives a standard eigenvalue problem with eigenvalues $\lambda=\omega^{2}$ indicating the frequencies at which a wave can propagate in the structure when a given phase is specified between the edges of the cell. It is noted that in this work, only the real part of the eigenvalues is examined since the target of the examination is the band gap behaviour.

\subsection{Optimisation}

The optimisation method (see Fig. 3) that is employed to calculate the optimal structure design is described below. The set of parameters can be expressed as

$$
\mathbf{p}=\left\{\beta_{1}, \beta_{2}, \ldots, \beta_{n}\right\},
$$

where $n$ is the number of parameters examined. The design values may be considered to be constrained (e.g. $\beta_{i} \in\left[\beta_{i, \min }, \beta_{i, \max }\right]$ ). The considered variables are the mass $m$, the bending stiffness $b_{-} s$, the band gap's width $b_{-} g$ and the midpoint frequency of the band gap $b_{-} g_{m_{-} f}$ (aiming for band gaps at frequencies as low as possible) of the stiffened plate. Examples of parameters $\beta_{i}$ would be any 


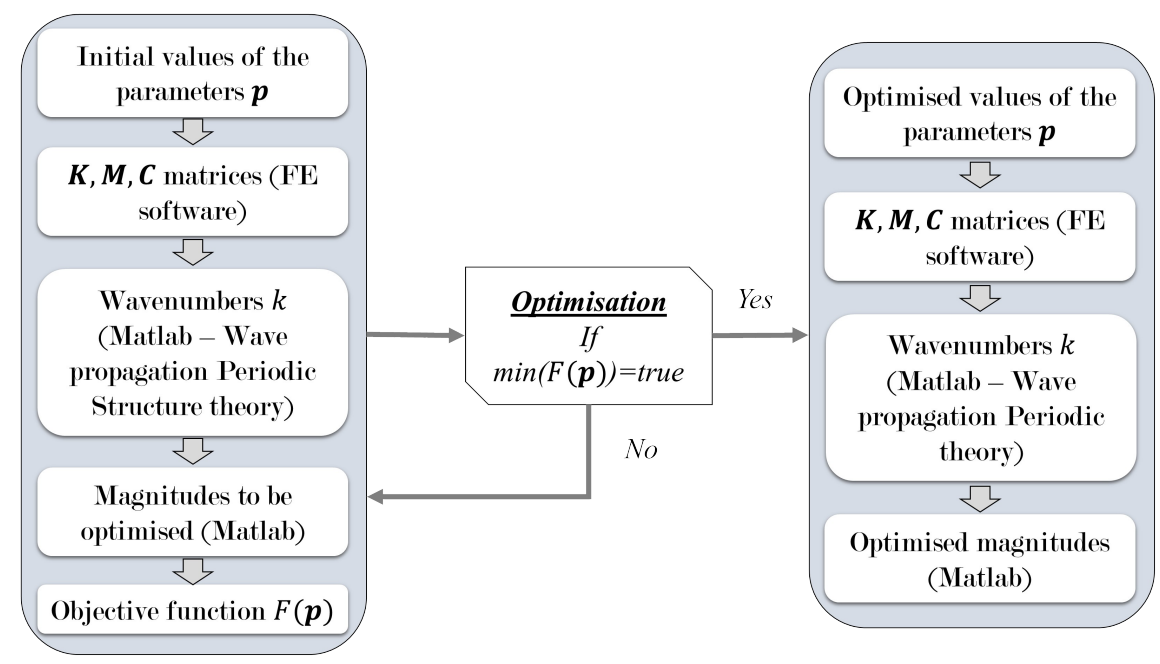

Figure 3: Flow chart of the optimisation method.

geometrical characteristic of the stiffener or the plate itself, such as thickness, that affects the considered variables. The objective function for the specific structure is

$$
\begin{aligned}
F(\mathbf{p})= & a_{3} \times m^{3}+a_{2} \times m^{2}+a_{1} \times m+a_{0}+b_{3} \times b_{-} s^{3}+b_{2} \times b_{-} s^{2}+b_{1} \times b_{-} s+b_{0} \\
& +c_{3} \times b_{-} g^{3}+c_{2} \times b_{-} g^{2}+c_{1} \times b_{-} g+c_{0}+d_{3} \times b_{-} g_{m_{-} f}^{3}+d_{2} \times b_{-} g_{m_{-} f}^{2}+ \\
& d_{1} \times b_{-} g_{m_{-} f}+d_{0}
\end{aligned}
$$

where $a_{i}, b_{i}, c_{i}$ and $d_{i}$ are the coefficients given by the design cost functions. Adjusting these coefficients, the designers are given the ability to apply polynomial curves to available cost data forming the cost functions of the optimisation, as can be seen in the next section (see Fig. 5). Additionally, this type of objective function renders its differentiability and the calculation of its partial gradients efficient. Higher order polynomial or exponential fitting functions may be applied without loss of accuracy. The gradient of $F(\mathbf{p})$ can, therefore, be computed as

$$
\nabla F(\mathbf{p})=\left\{\frac{\partial F(\mathbf{p})}{\partial \beta_{i}}\right\}^{T}
$$


By definition, we have $b_{-} g=f_{2}-f_{1}, b_{-} g_{m_{-} f}=\frac{f_{1}+f_{2}}{2}$ and $f_{i}=\frac{\omega_{i}}{2 \pi}, \lambda_{i}=\omega_{i}^{2}$, where $f_{1}$ and $f_{2}$ are the frequencies at the lower and upper bound of the band gap. Thus,

$$
\begin{gathered}
\frac{\partial b_{-} g}{\partial \beta_{i}}=\frac{\partial f_{2}}{\partial \beta_{i}}-\frac{\partial f_{1}}{\partial \beta_{i}}=\frac{1}{2 \pi} \frac{\partial \omega_{2}}{\partial \beta_{i}}-\frac{1}{2 \pi} \frac{\partial \omega_{1}}{\partial \beta_{i}}=\frac{1}{2 \pi}\left(\frac{\partial \sqrt{\lambda_{2}}}{\partial \beta_{i}}-\frac{\partial \sqrt{\lambda_{1}}}{\partial \beta_{i}}\right), \\
\frac{\partial b_{-} g_{m_{-} f}}{\partial \beta_{i}}=\frac{1}{2}\left(\frac{\partial f_{1}}{\partial \beta_{i}}+\frac{\partial f_{2}}{\partial \beta_{i}}\right)=\frac{1}{4 \pi}\left(\frac{\partial \omega_{1}}{\partial \beta_{i}}+\frac{\partial \omega_{2}}{\partial \beta_{i}}\right)=\frac{1}{4 \pi}\left(\frac{\partial \sqrt{\lambda_{1}}}{\partial \beta_{i}}+\frac{\partial \sqrt{\lambda_{2}}}{\partial \beta_{i}}\right) .
\end{gathered}
$$

Taking advantage of the chain rule of differentiation, we get:

$$
\frac{\partial \sqrt{\lambda_{i}}}{\partial \beta_{i}}=\frac{\partial \sqrt{\lambda_{i}}}{\partial \lambda_{i}} \frac{\partial \lambda_{i}}{\partial \beta_{i}}=\frac{1}{2 \sqrt{\lambda_{i}}} \frac{\partial \lambda_{i}}{\partial \beta_{i}}
$$

where the partial derivatives of the eigenvalues are calculated based on the following techniques, given elsewhere [39, 40]:

$$
\frac{\partial \lambda_{i}}{\partial \beta_{i}}=\frac{-\mathbf{x}^{T}\left(\lambda_{i}^{2} \frac{\partial \mathbf{M}}{\partial \beta_{i}}+\lambda_{i} \frac{\partial \mathbf{C}}{\partial \beta_{i}}+\frac{\partial \mathbf{K}}{\partial \beta_{i}}\right) \mathbf{x}}{\mathbf{x}^{T}\left(2 \lambda_{i} \mathbf{M}+\mathbf{C}\right) \mathbf{x}}
$$

where $\mathbf{x}=\mathbf{q}^{\prime}=\left[\mathbf{q}_{I} \mathbf{q}_{L}\right]^{T}$ is the eigenvector. In this work, the partial derivatives of $\mathbf{K}, \mathbf{M}$ and $\mathbf{C}$ are calculated considering a perturbation of $0.1 \%$ of the examined parameter $\beta_{i}$, a percentage that was the result of a convergence analysis. Thus, we have:

$$
\frac{\partial \mathbf{K}}{\partial \beta_{i}}=\frac{\mathbf{K}_{p}-\mathbf{K}_{0}}{\beta_{i, p}-\beta_{i, 0}}, \quad \frac{\partial \mathbf{M}}{\partial \beta_{i}}=\frac{\mathbf{M}_{p}-\mathbf{M}_{0}}{\beta_{i, p}-\beta_{i, 0}}, \quad \frac{\partial \mathbf{C}}{\partial \beta_{i}}=\frac{\mathbf{C}_{p}-\mathbf{C}_{0}}{\beta_{i, p}-\beta_{i, 0}},
$$

where $\mathbf{K}_{p}, \mathbf{M}_{p}$ and $\mathbf{C}_{p}$ are the perturbed stiffness, mass and damping matrices, $\beta_{i, p}$ is the perturbed considered parameter and $\beta_{i, 0}$ the unperturbed parameter. The mass, stiffness and damping matrices $\mathbf{M}, \mathbf{K}$ and $\mathbf{C}$ of the unit cell can also be formed by assembling the local mass, stiffness and damping matrices of individual FEs, which can be calculated analytically [41]. It is, therefore, evident that when the expressions of the partial derivatives for every local mass, stiffness and damping matrix $\frac{\partial \mathbf{m}}{\partial \beta_{i}}, \frac{\partial \mathbf{k}}{\partial \beta_{i}}$ and $\frac{\partial \mathbf{c}}{\partial \beta_{i}}$ are known, then the expressions for the global matrices $\frac{\partial \mathbf{M}}{\partial \beta_{i}}, \frac{\partial \mathbf{K}}{\partial \beta_{i}}$ and $\frac{\partial \mathbf{C}}{\partial \beta_{i}}$ can be derived simply by assembling 
the expressions of the local matrices together. Therefore, we have:

$$
\frac{\partial \lambda_{i}}{\partial \beta_{i}}=\frac{-\mathbf{x}^{T}\left(\lambda_{i}^{2} \mathbf{R}^{H} \frac{\partial \mathbf{M}}{\partial \beta_{i}} \mathbf{R}+\lambda_{i} \mathbf{R}^{H} \frac{\partial \mathbf{C}}{\partial \beta_{i}} \mathbf{R}+\mathbf{R}^{H} \frac{\partial \mathbf{K}}{\partial \beta_{i}} \mathbf{R}\right) \mathbf{x}}{\mathbf{x}^{T}\left(2 \lambda_{i} \mathbf{R}^{H} \mathbf{M} \mathbf{R}+\mathbf{R}^{H} \mathbf{C R}\right) \mathbf{x}} .
$$

The calculation of $\frac{\partial b_{-} s}{\partial \beta_{i}}, \frac{\partial m}{\partial \beta_{i}}$ depends on the geometric and material properties of the case study for which the optimisation method is applied. A constrained nonlinear interior point optimisation algorithm using Newton's method [42] is employed to compute the optimal parameter vector $\mathbf{p}$ that minimises $F(\mathbf{p})$. It should be noted that the optimisation used in this work leads to results in significantly shorter times compared to those that examine the whole finite periodic structure, since it only needs the unit cell's wave propagation characteristics. Furthermore, the adaptability of the optimisation method allows the examination of both types of band gap mechanism (Bragg scattering and local resonance). On the other hand, when boundary conditions are applied to the structure PST fails to include their effects on the dynamic and stop band behaviour.

\section{Case study}

The band gap behaviour of a thin composite beam with AM stiffener is examined and optimised using the scheme described in section 2.2. In this case, the Bragg scattering mechanism is targeted and the absolute band gap is examined. A thin plate of carbon fibre is used as a substrate structure and the stiffener is manufactured using the powder bed fusion method and PA12 material with an EOS Formiga P100 machine. The stiffener is cemented to the plate using a twopart epoxy adhesive (Permabond ET500). The parameters that are examined are the thickness of the stiffener $t_{s t}$ and the length of the top of the wedge-like shape of the stiffener $l_{t o p}$ (see Table 1 and Fig. 4). The mechanical properties of the materials are given in Table 2. Structural damping of both materials (loss factor $\eta$ ) is modelled. Since the host structure's characteristics are constant, only the stiffeners' design variables $m$ and $b \_s$ are investigated. 


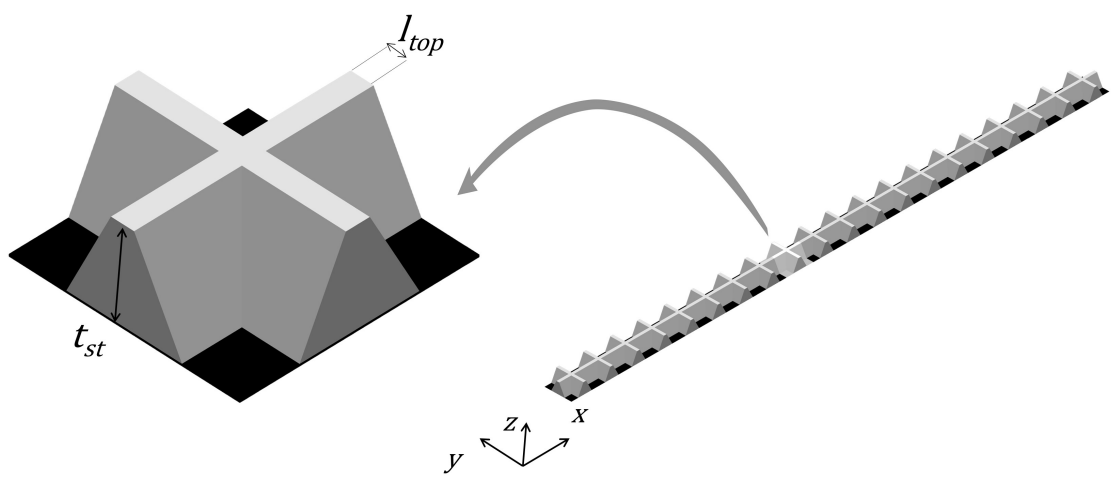

Figure 4: Schema of periodic beam and unit cell.

Table 1: Case study optimisation variables and parameters.

\begin{tabular}{cc}
\hline \hline Optimised variables & Parameters \\
\hline Mass, $m$ & Length of the top side, $l_{t o p}$ \\
Bending stiffness, $b_{-} s$ & Thickness of the stiffener, $t_{s t}$ \\
Band gap's width, $b_{-} g$ & \\
Band gap's mid-point frequency, $b_{-} g_{m_{-} f}$ & \\
\hline
\end{tabular}

\subsection{Numerical results}

The examined parameters are constrained such that $l_{t o p} \in[0.005 \mathrm{~m}, 0.025 \mathrm{~m}]$ and $t_{s t} \in[0.005 \mathrm{~m}, 0.020 \mathrm{~m}]$. In Appendix A, the calculations of $\frac{\partial b_{-} s}{\partial \beta_{i}}, \frac{\partial m}{\partial \beta_{i}}$ are given. The FE model of the unit cell consists of 468 8-node hexahedral elements (144 of which are used to model the carbon fibre strip and 324 the stiffener). The design cost functions employed to inform the relationship between $b_{-} g, b_{-} g_{m_{-} f}, m$ and $b \_s$ and the induced design cost, are shown in Fig. 5. The cost functions are based on the aim of a lightweight structural design with a wide band gap at low frequencies. The bending stiffness cost curve is chosen bearing in mind that while large values of stiffness are desired, values that are too high may contradict 

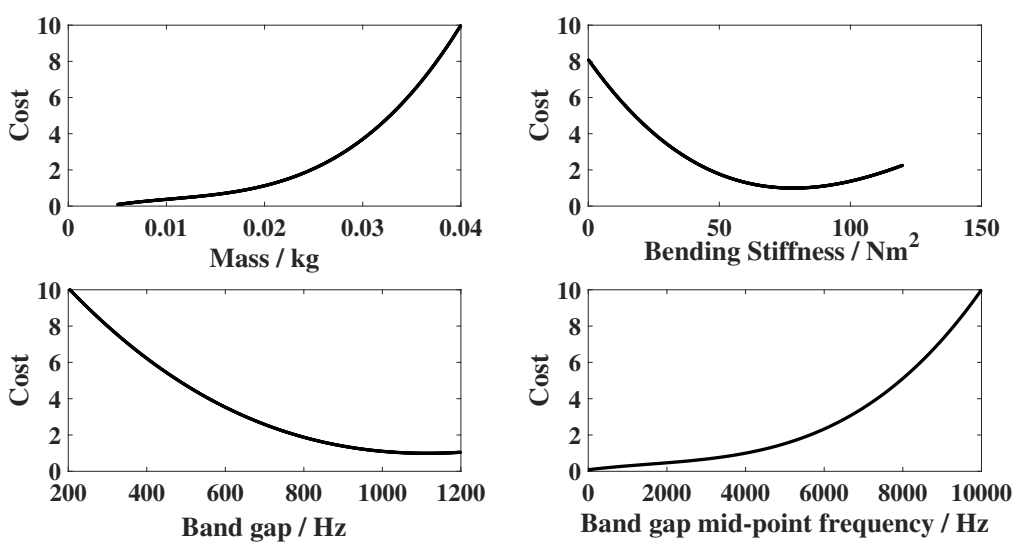

Figure 5: Cost functions used in the optimisation method.

Table 2: Material properties

\begin{tabular}{cc}
\hline \hline Cured Carbon Fibre & PA12 \\
\hline$\rho=1870 \mathrm{~kg} / \mathrm{m}^{3}$ & $\rho=900 \mathrm{~kg} / \mathrm{m}^{3}$ \\
$E_{x}=40 \mathrm{GPa}$ & $E=1600 \mathrm{MPa}$ \\
$E_{y}=40 \mathrm{GPa}$ & $\nu=0.3$ \\
$E_{z}=5 \mathrm{GPa}$ & $\eta=4 \%$ \\
$\nu_{x y}=0.4$ & \\
$\nu_{y z}=0.4$ & \\
$\nu_{x z}=0.25$ & \\
$G_{x y}=1.2 \mathrm{GPa}$ & \\
$G_{y z}=1.2 \mathrm{GPa}$ & \\
$G_{x z}=3.6 \mathrm{GPa}$ & \\
$\eta$ & $=1 \%$ \\
\hline
\end{tabular}

the aims of the final design of the structure. Nevertheless, it should be noted that the main aim of this work is to provide an optimisation tool which can be adapted to different applications and the cost functions are arbitrarily chosen. When the method is tailored for a specific application, the cost functions can be formulated by the designers accordingly. In this case, the cost functions chosen 


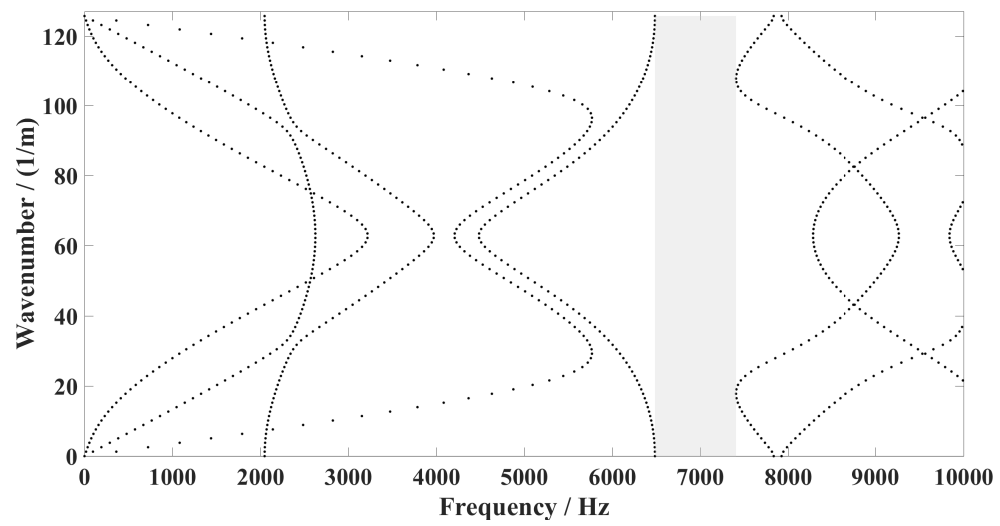

Figure 6: Wave numbers of the numerical analysis of the beam with geometric characteristics given by the optimal parameters. The shaded area represents the band gap.

for the examined structure lead to the objective function (SI units are used during the optimisation):

$$
\begin{aligned}
F(\mathbf{p})= & 3.167 \times 10^{5} \times m^{3}-9.890 \times 10^{3} \times m^{2}+1.503 \times 10^{2} \times m-0.4573 \\
& -3.701 \times 10^{-6} \times b_{-} s^{3}+1.743 \times 10^{-3} \times b_{-} s^{2}-2.046 \times 10^{-1} \times b_{-} s \\
& +8.102-3.214 \times 10^{-9} \times b_{-} g^{3}+1.870 \times 10^{-5} \times b_{-} g^{2} \\
& -2.970 \times 10^{-2} \times b_{-} g+15.314+1.358 \times 10^{-11} \times b_{-} g_{m_{-} f}^{3} \\
& -6.296 \times 10^{-8} \times b_{-} g_{m_{-} f}^{2}+2.630 \times 10^{-4} \times b_{-} g_{m_{-} f}+8.642 \times 10^{-2},
\end{aligned}
$$

where the gradient of $F(\mathbf{p})$ is

$$
\nabla F(\mathbf{p})=\left\{\frac{\partial F(\mathbf{p})}{\partial t_{s t}} \frac{\partial F(\mathbf{p})}{\partial l_{t o p}}\right\}^{T}
$$

145 and together with the parametric analysis results we consider this as the optimal design (see Fig. 8). The optimal values of the parameters are found to be: $l_{\text {top }}=5 \mathrm{~mm}$ and $t_{s t}=19.8 \mathrm{~mm}$, which gives: $b_{-} g=920 \mathrm{~Hz}, b_{-} g_{m_{-} f}=6950 \mathrm{~Hz}$, $m=24.4 \mathrm{~g}$ and $b \_s=44 \mathrm{Nm}^{2}$ (of the stiffener). The propagating wave numbers 150 observed. 


\subsection{Parametric analysis of the hybrid structure}
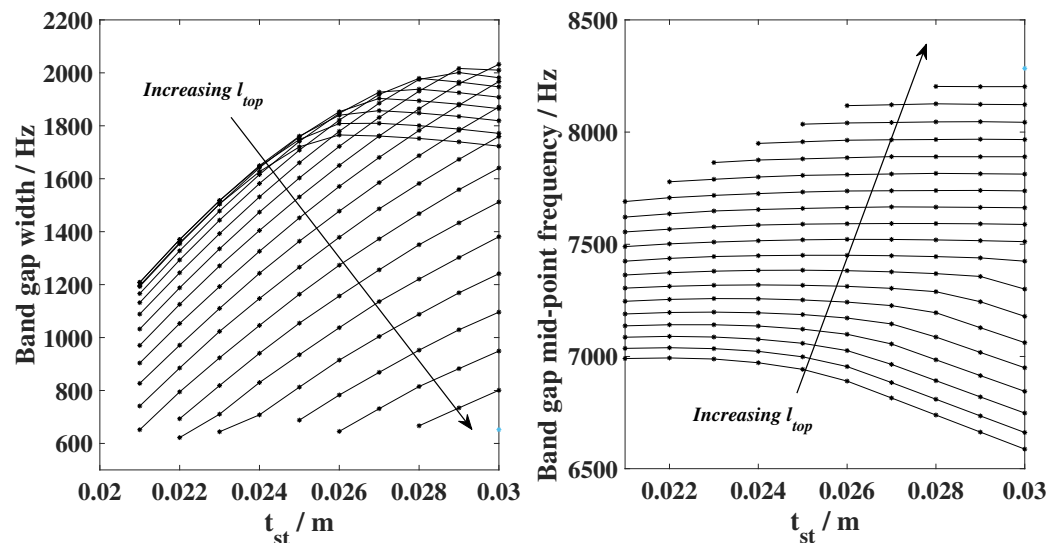

Figure 7: Parametric analysis results of further cases with $t_{s t}$ out of the optimisation limits, $t_{s t} \in[0.021 \mathrm{~m}, 0.030 \mathrm{~m}]$ and $l_{\text {top }} \in[0.005 \mathrm{~m}, 0.025 \mathrm{~m}]$.

A parametric analysis is performed to examine the effect of the two design

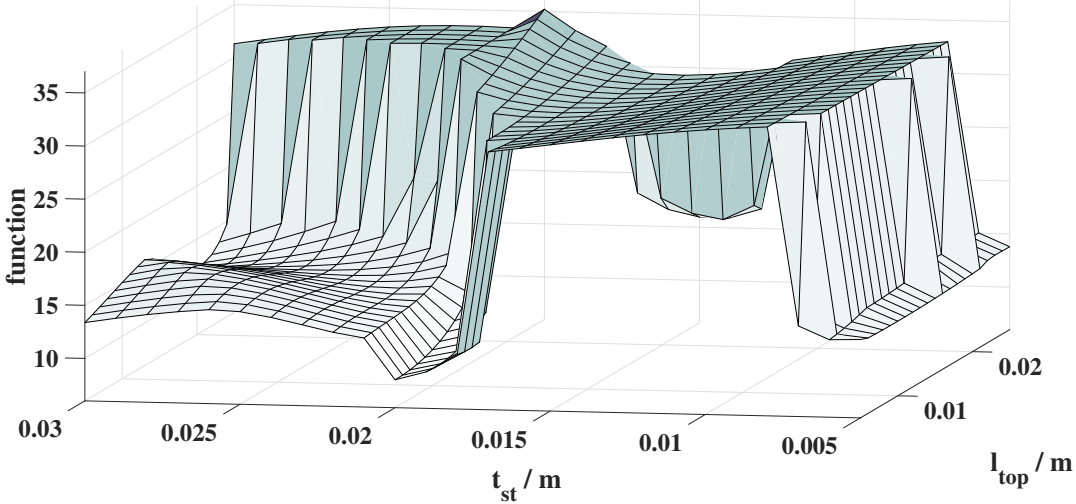

Figure 8: Objective function of the optimisation as calculated using the parametric analysis results $\left(t_{s t} \in[0.005 \mathrm{~m}, 0.030 \mathrm{~m}]\right.$ and $\left.l_{\text {top }} \in[0.005 \mathrm{~m}, 0.025 \mathrm{~m}]\right)$.

variables on the band gap behaviour of the structure. For this reason, several solutions are acquired between the optimisation procedure limits $\left(l_{t o p} \in\right.$ $[0.005 \mathrm{~m}, 0.025 \mathrm{~m}]$ and $\left.t_{s t} \in[0.005 \mathrm{~m}, 0.020 \mathrm{~m}]\right)$, with a step of $0.001 \mathrm{~m}$ between 

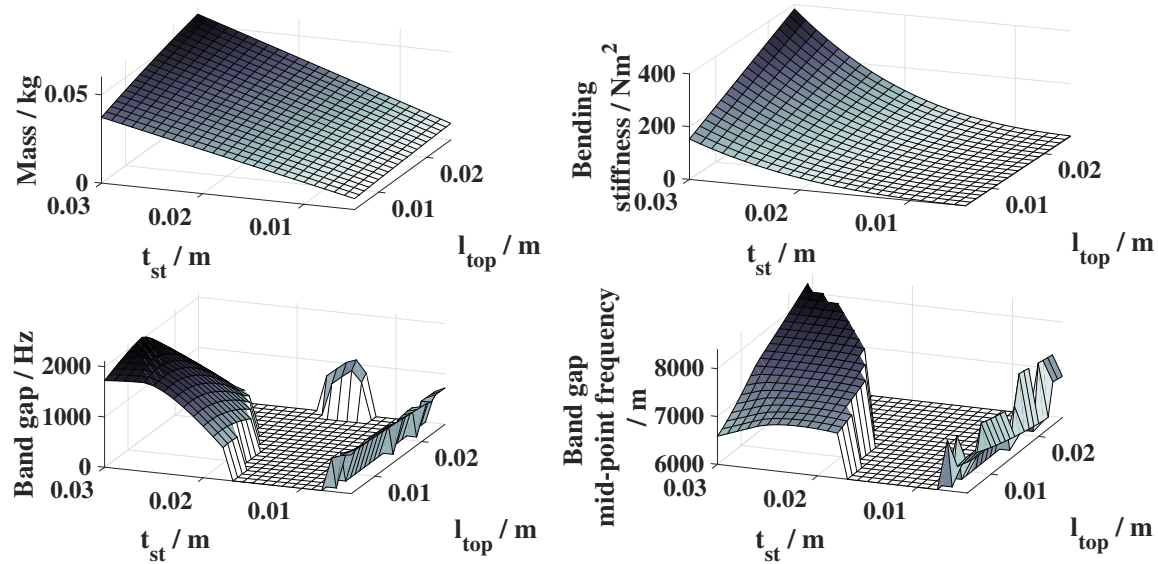

Figure 9: Relation of the optimised variables $m, b_{-} s, b_{-} g$ and $b_{-} g_{m_{-} f}$ with regards to parameters $t_{\text {st }}$ and $l_{\text {top }}\left(t_{\text {st }} \in[0.005 \mathrm{~m}, 0.030 \mathrm{~m}]\right.$ and $\left.l_{\text {top }} \in[0.005 \mathrm{~m}, 0.025 \mathrm{~m}]\right)$.

the solutions. It is observed that between $t_{s t}=0.008 \mathrm{~m}$ and $t_{s t}=0.017 \mathrm{~m}$, there is no significant band gap $\left(b_{-} g<200 \mathrm{~Hz}\right)$. In view of this observation, a further parametric analysis is completed; this time examining design variables $t_{s t} \in[0.021 \mathrm{~m}, 0.030 \mathrm{~m}]$ and $l_{t o p} \in[0.005 \mathrm{~m}, 0.025 \mathrm{~m}]$. In Fig. 7, it is shown that for a given $t_{s t}$, as $l_{t o p}$ is increased, the band gap mid-point frequency is increased; however, the band gap width gets smaller. On the other hand, for a given $l_{t o p}$, we see the band gap increasing and its mid-point frequency staying almost constant; but this behaviour changes after a specific value of $t_{s t}$ (which varies) and both band gap, and its mid-point frequency, decreases. Having acquired full solutions in parametric analysis, a graph of the cost function's values (Eq. (25)) is generated (see Fig. 8). It is observed that the result of the optimisation lies on the area that includes the global optimum. It is noted that the values of the objective functions that are larger than 20 are the cases for which a very narrow band gap is observed. This becomes apparent in Fig. 9, where the relations between the optimised variables $m, b_{-} s, b_{-} g$ and $b_{-} g_{m_{-} f}$ with regards to parameters $t_{s t}$ and $l_{t o p}$ are presented. In these graphs, it can also be observed that there are several combinations of $t_{s t}$ and $l_{t o p}$ which lead to significantly wider band gaps at lower frequencies but with much higher mass, compromis- 
ing the objective function outcome. This behaviour highlights the significance of the cost functions and the designers' input during the design of a structure which may aim for higher band gap without penalising the additional mass required. Furthermore, additional options can be added to the methodology so that Pareto fronts are calculated, offering the designers numerous alternative solutions.

\section{Finite element analysis and experimental results}

FE harmonic analysis is undertaken using the same FE model as that for the unit cell of the wave propagation analysis and examining three different lengths of the hybrid beam. Previous research on bang gap behaviour of periodic structures has shown that the effect of the number of the unit cells must be considered. Jensen [43] showed analytically that the level of vibration attenuation depends on the number of unit cells; an effect that was also demonstrated experimentally elsewhere [44]. More specifically, it was shown that the band gap becomes more distinguishable (meaning the vibration attenuation is higher) as the number of unit cell repetitions increases. To check this behaviour in the specific case study, FE models with 468 8-node hexahedral elements per unit cell and for three different cases are examined: 5-cell, 10-cell and 15-cell beams. In Fig. 10, a representation of the simulation of the experiment for the 5-cell case is shown, where the excitation and response locations can be seen (no boundaries were applied). Similar conditions are used for the 10- and 15-cell case simulations, with the excitation force always applied on one edge of the carbon fibre strip and the response is measured on the top of the stiffener, on the other end. The results are shown in Fig. 11, where the effect of the number of the unit cells on the vibration attenuation of the band gap can be observed.

The geometry resulting from the optimisation process is manufactured and dynamically tested (Fig. 12) to validate the FE analysis results. A 5-cell beam is preferred, due to the limitation of the mass of the specimen that the shaker can excite and on the AM machine production size. It is noted that the exper- 


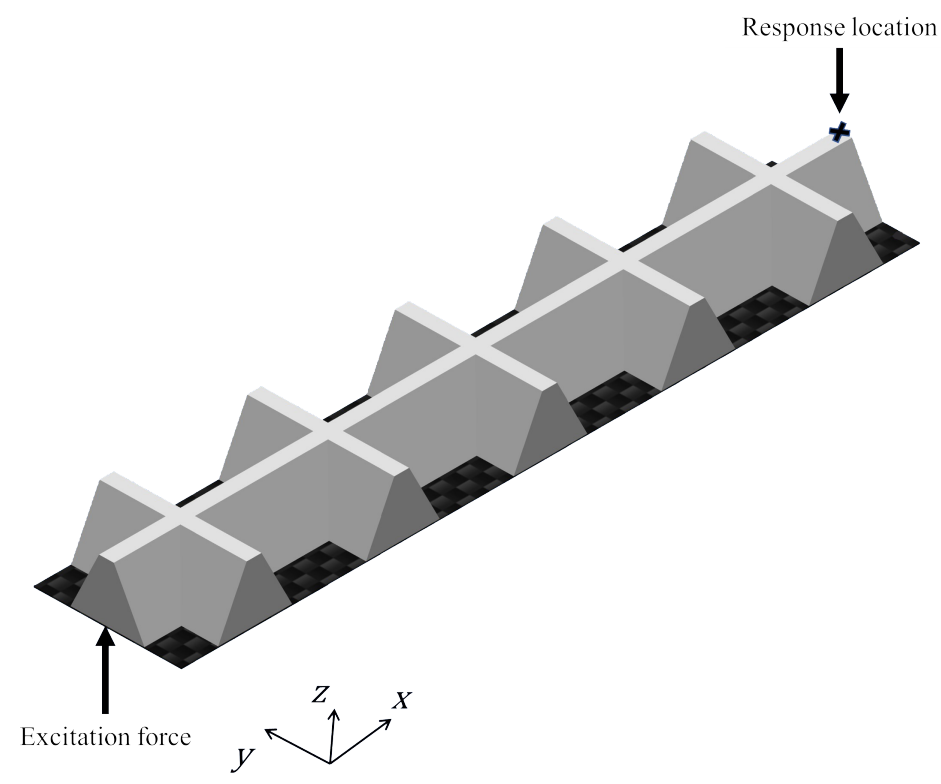

Figure 10: Representation of simulation of the experiment.

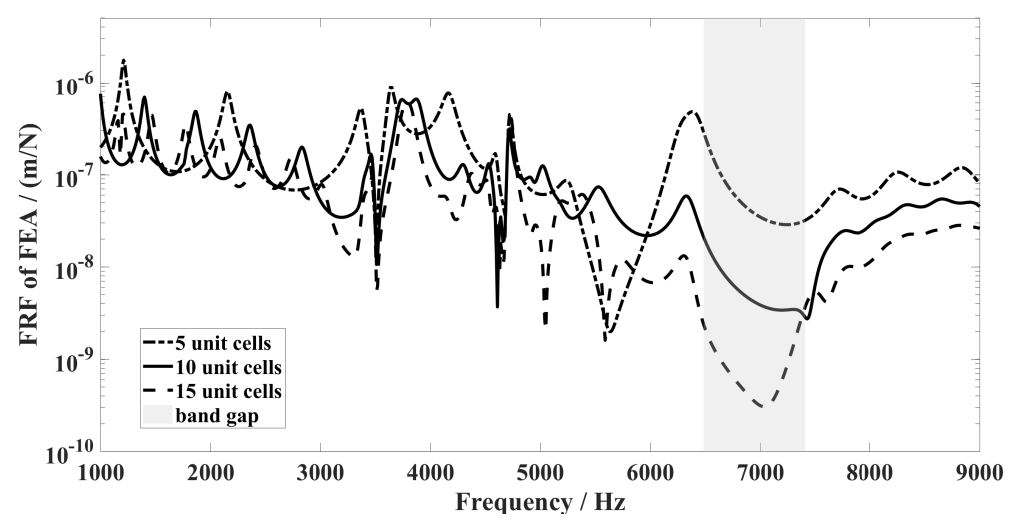

Figure 11: FRF of FEA of hybrid composite beam with: (-.) 5 unit cells, (-) 10 unit cells, (-) 15 unit cells. The shaded area represents the numerically calculated band gap.

imental results do not include the band gap observation due to an insufficient number of the unit cells. An electrodynamic shaker (TBS K2007E1) is used to excite the specimen between $1 \mathrm{kHz}$ and $8.5 \mathrm{kHz}$, with the signal produced and 
analysed by a Polytec VIB-E-400 Junction Box using a fast Fourier transform (FFT). A single point laser vibrometer (Polytec PDV-100) is used to measure the displacement at several points on the specimen (Fig. 13). An impedance head (PCB 288D01) is mounted on one edge of the specimen and provides the measurement of the input force on the mounting point of the specimen. The impedance head is then bolted on to the moving head of the shaker. The shaker is mounted on an aluminium rig, designed specifically for vibration experiments. As can be seen in Fig. 13, the points' number is indicative of the unit cell's repetition.
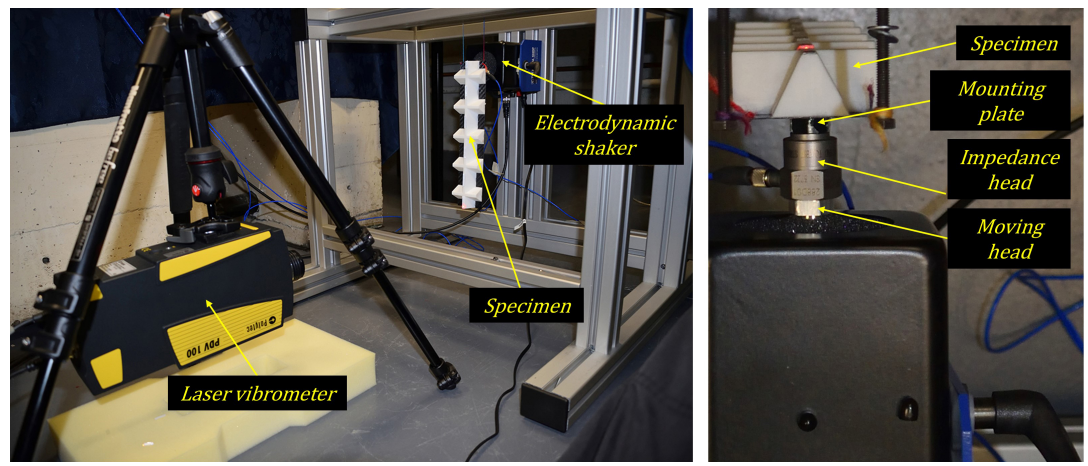

Figure 12: Experimental setup of the vibration testing.

In Fig. 14 to Fig. 16, the results for several points along $x$-axis are shown. The frequency response function (FRF) graphs of the experimental and FE results of the three points show convergence, with minor deviations in some high frequency resonant modes. Point 1 (Fig. 14) has the most significant deviations, while point 3 (Fig. 15) and point 5 (Fig. 16) exhibit better convergence with the FE results. The highlight of the experimental results is the absence of resonant modes in the calculated band gap area, as it is expected from a band gap structure. Nevertheless, as discussed above, 5 cells are not sufficient to make the vibration attenuation distinguishable in this frequency band. 

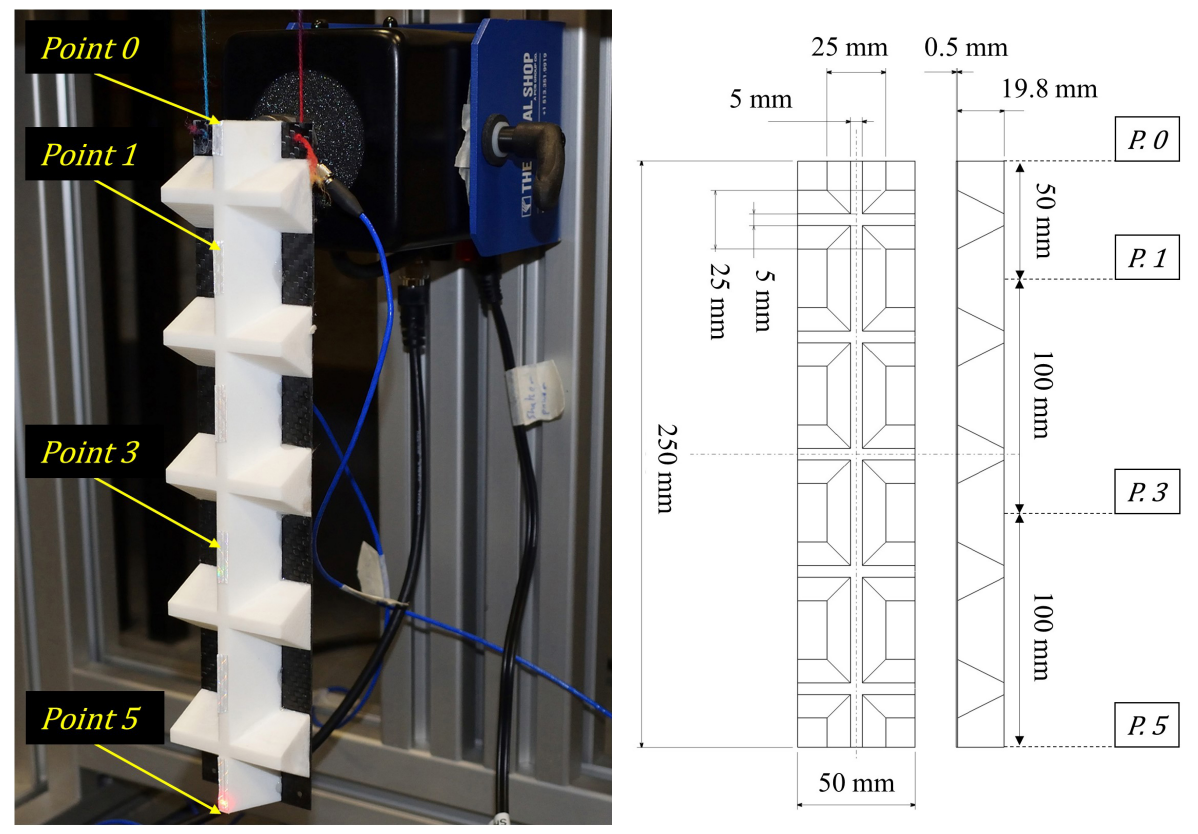

Figure 13: The points at which the FRF graphs are presented.

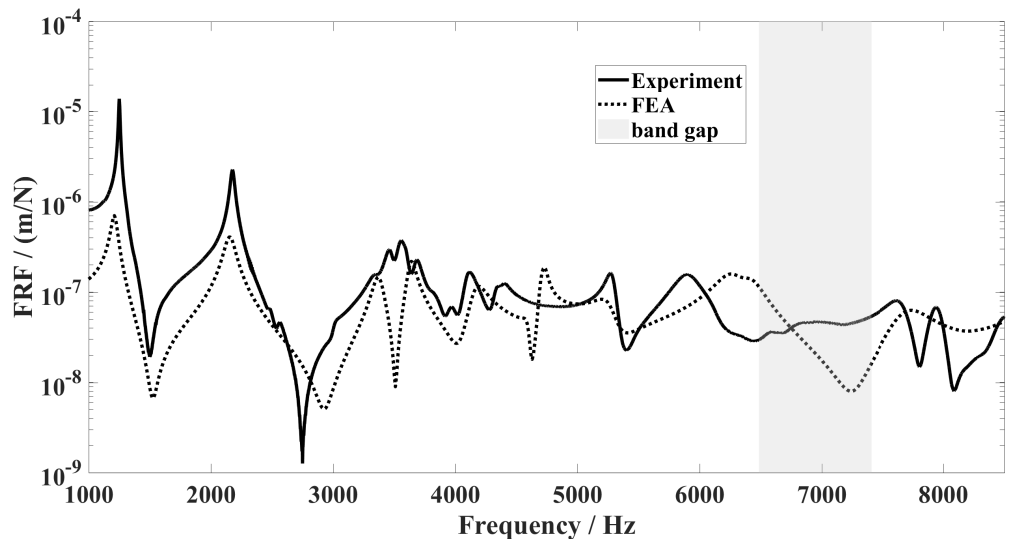

Figure 14: Experimental and FEA results of the FRF of point 1 where $(-)$ is FRF of experimental results and (- -) is the FEA results. 


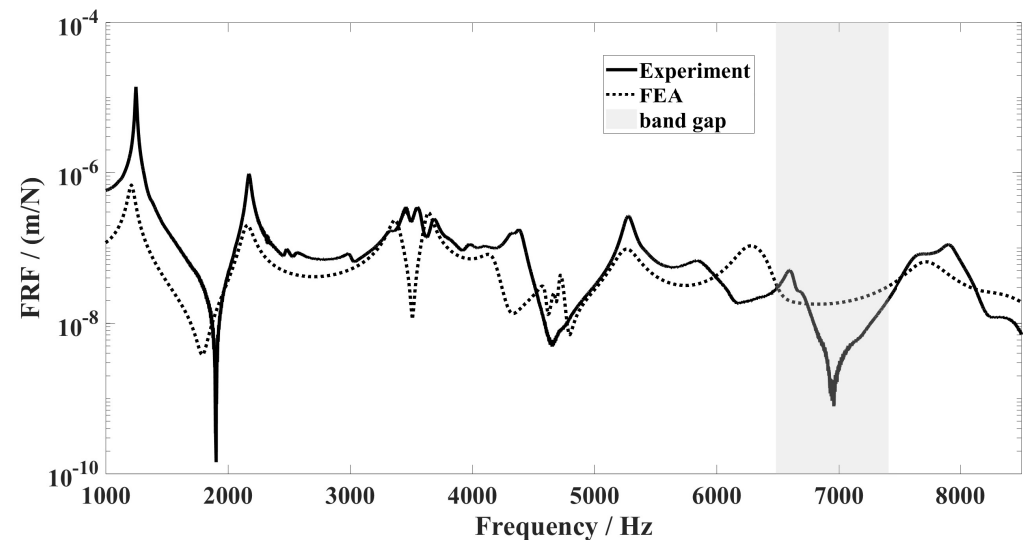

Figure 15: Experimental and FEA results of the FRF of point 3 where $(-)$ is FRF of experimental results and (- -) is the FEA results.

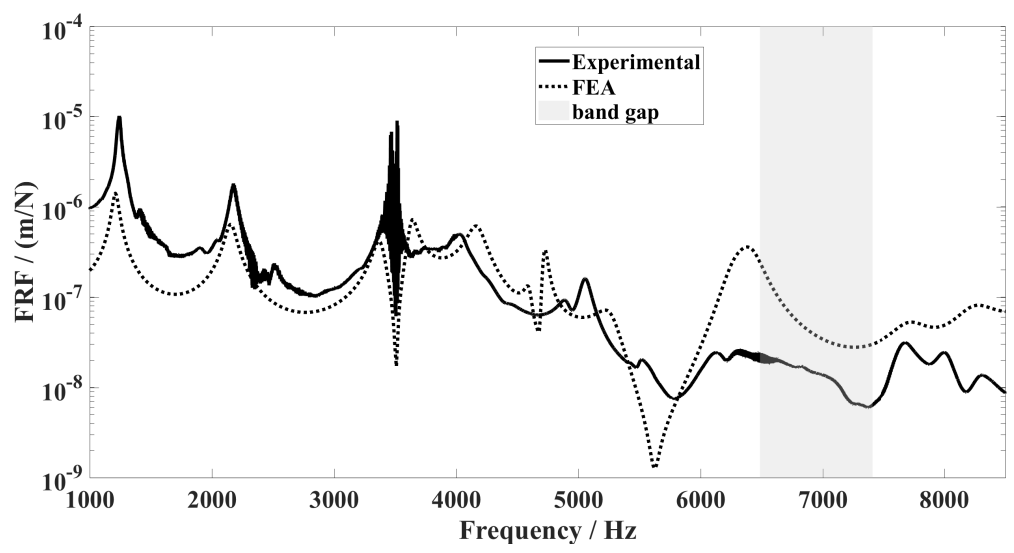

Figure 16: Experimental and FEA results of the FRF of point 5 where $(-)$ is FRF of experimental results and (- - is the FEA results..

\section{Concluding remarks}

In this work, an efficient multi-objective scheme using scalarisation is presented for optimising the static, dynamic and mass characteristics of a onedimensional composite structure having an additive manufactured stiffener. Additionally, an experimental evaluation of the FE model of the case study is 
presented. The presented computational scheme gives the ability to adapt the optimisation criteria to specific applications. Furthermore, a parametric analysis is completed offering a valuable insight of the stop band behaviour of the structure with regards to the parameters.

\section{Acknowledgements}

This research is supported by the European Union's Horizon 2020 funded grant 'SAFE-FLY', under the Marie Skłodowska-Curie grant agreement No. 721455 and the Engineering and Physical Sciences Research Council funded grant 'Metrology for precision and additive manufacturing' (EP/M008983/1). The authors are grateful for the help and time of Dr Wahyudin P. Syam (University of Nottingham) in the experimental part of the work.

\section{Appendix A. Optimisation calculations}
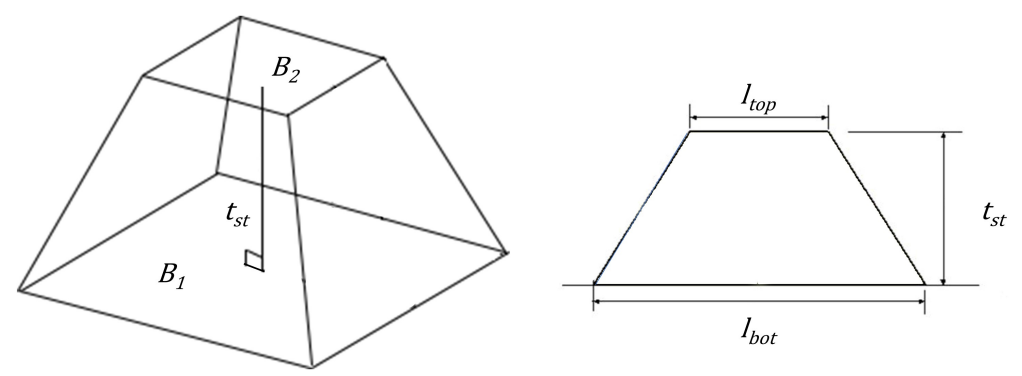

Figure Appendix A.1: Pyramid frustum (left) and trapezoid (right).

Further calculations used in the optimisation of the band gap behaviour of the beam are presented in this section. For the calculation of the mass 
$m=m_{s t}$ the formulae for volume calculation of a frustum of a pyramid and of a trapezoidal structure are used, so that $m_{s t}=\rho_{P A 12}\left(2 V_{t r}-V_{f r}\right)$, where $V_{t r}=L_{x}\left(l_{\text {bot }}+l_{t o p}\right) \frac{t_{s t}}{2}$ and $V_{f r}=\frac{t_{s t}}{3}\left(B_{1}+B_{2}+\sqrt{B_{1} B_{2}}\right)$ (see Fig. Appendix A.1 for labels, were $B_{1}$ and $B_{2}$ are the bottom and top areas of the frustum, respectively). The mass of the stiffener $m_{s t}$ and its partial derivatives in terms of the parameters, $t_{s t}$ and $l_{t o p}$, are calculated as follows:

$$
\begin{gathered}
m_{s t}=t_{s t} \rho_{p}\left[L_{x}\left(l_{\text {top }}+l_{\text {bot }}\right)-\frac{l_{\text {bot }}^{2}+l_{\text {top }}^{2}+l_{\text {bot }} l_{\text {top }}}{3}\right] \\
\frac{\partial m_{s t}}{\partial t_{s t}}=\rho_{p}\left[L_{x}\left(l_{\text {top }}+l_{\text {bot }}\right)-\frac{l_{b o t}^{2}+l_{\text {top }}^{2}+l_{\text {bot }} l_{\text {top }}}{3}\right] \\
\frac{\partial m_{s t}}{\partial l_{\text {top }}}=\rho_{p} t_{s t}\left[L_{x}-\frac{2 l_{t o p}+l_{b o t}}{3}\right] .
\end{gathered}
$$

The bending stiffness of the stiffener $b_{-} s$, along with its partial derivatives are given of the following equations:

$$
\begin{gathered}
b_{-} s_{s t}=E_{p} t_{s t}^{3} \frac{3 l_{t o p}+l_{\text {bot }}}{12}, \\
\frac{\partial b_{\_} s}{\partial t_{s t}}=E_{p} t_{s t}^{2} \frac{3 l_{t o p}+l_{b o t}}{4}, \quad \frac{\partial b_{-} s}{\partial l_{\text {top }}}=\frac{E_{p} t_{s t}^{3}}{4} .
\end{gathered}
$$

\section{Appendix B. Undamped system}

The homogenous equation in the reduced set of degrees of freedom for undamped system is given by

$$
\left[\mathbf{K}^{\prime}-\omega^{2} \mathbf{M}^{\prime}\right] \mathbf{q}=\mathbf{f}
$$

The partial derivative of the eigenvalue is

$$
\frac{\partial \lambda_{i}}{\partial \beta_{i}}=\mathbf{x}^{T}\left(\frac{\partial \mathbf{K}}{\partial \beta_{i}}-\lambda_{i} \frac{\partial \mathbf{M}}{\partial \beta_{i}}\right) \mathbf{x} .
$$

For the global matrices we have:

$$
\frac{\partial \lambda_{i}}{\partial \beta_{i}}=\mathbf{x}^{T}\left(\mathbf{R}^{H} \frac{\partial \mathbf{K}}{\partial \beta_{i}} \mathbf{R}-\lambda_{i} \mathbf{R}^{H} \frac{\partial \mathbf{M}}{\partial \beta_{i}} \mathbf{R}\right) \mathbf{x} .
$$




\section{References}

[1] G. Floquet, Sur les equations differentielles lineaires, Ann. ENS [2] 12 (1883) (1883) 47-88.

[2] L. Rayleigh, Xvii. on the maintenance of vibrations by forces of double frequency, and on the propagation of waves through a medium endowed with a periodic structure, The London, Edinburgh, and Dublin Philosophical Magazine and Journal of Science 24 (147) (1887) 145-159. doi:10.1080/14786448708628074.

[3] D. Mead, A general theory of harmonic wave propagation in linear periodic systems with multiple coupling, Journal of Sound and Vibration 27 (2) (1973) 235-260. doi:10.1016/0022-460X (73)90064-3.

[4] D. Mead, Wave propagation in continuous periodic structures: research contributions from southampton, 1964-1995, Journal of sound and vibration 190 (3) (1996) 495-524. doi:10.1006/jsvi.1996.0076.

[5] B. R. Mace, E. Manconi, Modelling wave propagation in two-dimensional structures using finite element analysis, Journal of Sound and Vibration 318 (4) (2008) 884-902. doi:10.1016/j.jsv.2008.04.039.

[6] V. Cotoni, R. Langley, P. Shorter, A statistical energy analysis subsystem formulation using finite element and periodic structure theory, Journal of Sound and Vibration 318 (4) (2008) 1077-1108. doi:10.1016/j.jsv. 2008.04.058.

[7] R. Langley, N. Bardell, P. Loasby, The optimal design of near-periodic structures to minimize vibration transmission and stress levels, Journal of Sound and Vibration 207 (5) (1997) 627-646. doi:10.1006/jsvi.1997. 1116.

[8] L. Brillouin, Wave propagation in periodic structures: electric filters and crystal lattices, McGrawHil, 1946. 
[9] E. Manconi, B. R. Mace, Estimation of the loss factor of viscoelastic laminated panels from finite element analysis, Journal of Sound and Vibration 329 (19) (2010) 3928-3939. doi:10.1016/j.jsv.2010.04.014.

[10] D. Chronopoulos, B. Troclet, O. Bareille, M. Ichchou, Modeling the response of composite panels by a dynamic stiffness approach, Composite Structures 96 (2013) 111-120. doi:10.1016/j.compstruct.2012.08.047.

[11] T. Ampatzidis, D. Chronopoulos, Acoustic transmission properties of pressurised and pre-stressed composite structures, Composite Structures 152 (2016) 900-912. doi:10.1016/j.compstruct.2016.06.027.

[12] I. Antoniadis, D. Chronopoulos, V. Spitas, D. Koulocheris, Hyper-damping properties of a stiff and stable linear oscillator with a negative stiffness element, Journal of Sound and Vibration 346 (2015) 37-52. doi:10.1016/ j.jsv.2015.02.028.

[13] D. Chronopoulos, I. Antoniadis, T. Ampatzidis, Enhanced acoustic insulation properties of composite metamaterials having embedded negative stiffness inclusions, Extreme Mechanics Lettersdoi:10.1016/j .eml. 2016. 10.012

[14] L. Van Belle, C. Claeys, E. Deckers, W. Desmet, On the impact of damping on the dispersion curves of a locally resonant metamaterial: Modelling and experimental validation, Journal of Sound and Vibration 409 (2017) 1-23. doi:10.1016/j.jsv.2017.07.045.

[15] C. Claeys, N. G. R. de Melo Filho, L. Van Belle, E. Deckers, W. Desmet, Design and validation of metamaterials for multiple structural stop bands in waveguides, Extreme Mechanics Letters 12 (2017) 7-22. doi:10.1016/ j.eml.2016.08.005.

[16] M. I. Hussein, M. J. Leamy, M. Ruzzene, Dynamics of phononic materials and structures: Historical origins, recent progress, and future outlook, Applied Mechanics Reviews 66 (4) (2014) 040802. doi:10.1115/1.4026911. 
[17] M. I. Hussein, G. M. Hulbert, R. A. Scott, Dispersive elastodynamics of 1d banded materials and structures: analysis, Journal of sound and vibration 289 (4) (2006) 779-806. doi:10.1016/j.jsv.2005.02.030.

[18] Z. Liu, X. Zhang, Y. Mao, Y. Zhu, Z. Yang, C. T. Chan, P. Sheng, Locally resonant sonic materials, Science 289 (5485) (2000) 1734-1736. doi:10. 1126/science.289.5485.1734.

[19] L. Liu, M. I. Hussein, Wave motion in periodic flexural beams and characterization of the transition between bragg scattering and local resonance, Journal of Applied Mechanics 79 (1) (2012) 011003. doi:10.1115/1. 4004592.

[20] J. Chen, C. Sun, Wave propagation in sandwich structures with resonators and periodic cores, Journal of Sandwich Structures \& Materials 15 (3) (2013) 359-374. doi:10.1177/1099636212468738.

[21] K. H. Matlack, A. Bauhofer, S. Krödel, A. Palermo, C. Daraio, Composite 3d-printed metastructures for low-frequency and broadband vibration absorption, Proceedings of the National Academy of Sciences (2016) 201600171doi:10.1073/pnas.1600171113.

[22] W. P. Syam, W. Jianwei, B. Zhao, I. Maskery, W. Elmadih, R. Leach, Design and analysis of strut-based lattice structures for vibration isolation, Precision Engineeringdoi:10.1016/j.precisioneng. 2017.09.010.

[23] A. Qureshi, B. Li, K. Tan, Numerical investigation of band gaps in 3d printed cantilever-in-mass metamaterials, Scientific reports 6 (2016) 28314. doi : 10.1038/srep28314.

[24] C. Claeys, E. Deckers, B. Pluymers, W. Desmet, A lightweight vibroacoustic metamaterial demonstrator: Numerical and experimental investigation, Mechanical Systems and Signal Processing 70 (2016) 853-880. doi:10.1016/j.ymssp.2015.08.029. 
[31] N. Olhoff, B. Niu, G. Cheng, Optimum design of band-gap beam structures, International Journal of Solids and Structures 49 (22) (2012) 3158-3169. doi:10.1016/j.ijsolstr.2012.06.014.

[32] M. I. Hussein, G. M. Hulbert, R. A. Scott, Dispersive elastodynamics of 1d 350

25] F. Warmuth, M. Wormser, C. Körner, Single phase 3d phononic band gap material, Scientific reports 7 (1) (2017) 3843. doi:10.1038/ s41598-017-04235-1.

[26] O. R. Bilal, A. Foehr, C. Daraio, Observation of trampoline phenomena in 3d-printed metamaterial plates, Extreme Mechanics Letters 15 (2017)

[27] M. Wormser, F. Wein, M. Stingl, C. Körner, Design and additive manufacturing of 3d phononic band gap structures based on gradient based optimization, Materials 10 (10) (2017) 1125. doi:10.3390/ma10101125.

[28] S. Taniker, C. Yilmaz, Design, analysis and experimental investigation of three-dimensional structures with inertial amplification induced vibration stop bands, International Journal of Solids and Structures 72 (2015) 88-97. doi:10.1016/j.ijsolstr.2015.07.013.

[29] A. Fabro, D. Beli, J. Arruda, N. Ferguson, B. Mace, Uncertainty analysis of band gaps for beams with periodically distributed resonators produced by additive manufacturing, in: ISMA 2016 Conference on Noise and Vibration Engineering, Belgium, 2016.

[30] D. Beli, J. Arruda, Influence of additive manufacturing variability in elastic band gaps of beams with periodically distributed resonators, in: Proceedings of the 3rd International Symposium on Uncertainty Quantification and Stochastic Modeling, 2016.

banded materials and structures: design, Journal of Sound and Vibration 307 (3) (2007) 865-893. doi:10.1016/j.jsv.2005.02.030. 
[33] M. I. Hussein, K. Hamza, G. M. Hulbert, R. A. Scott, K. Saitou, Multiobjective evolutionary optimization of periodic layered materials for desired wave dispersion characteristics, Structural and Multidisciplinary Optimization 31 (1) (2006) 60-75. doi:10.1007/s00158-005-0555-8.

[34] M. I. Hussein, K. Hamza, G. M. Hulbert, K. Saitou, Tailoring of twodimensional band-gap materials for broadband frequency isolation, in: ASME Conference Proceedings eds. Proceedings of the ASME 2007 International Design Engineering Technical Conferences \& Computers and Information in Engineering Conference. Las Vegas: ASME, 2007, pp. 485494. doi : 10.1115/DETC2007-35226.

[35] O. Sigmund, J. S. Jensen, Systematic design of phononic band-gap materials and structures by topology optimization, Philosophical Transactions of the Royal Society of London A: Mathematical, Physical and Engineering Sciences 361 (1806) (2003) 1001-1019. doi:10.1098/rsta.2003.1177.

[36] S. Neumark, Concept of complex stiffness applied to problems of oscillations with viscous and hysteretic damping, HM Stationery Office, 1962.

[37] S. Adhikari, Damping modelling using generalized proportional damping, Journal of Sound and Vibration 293 (1-2) (2006) 156-170. doi:10.1016/ j.jsv.2005.09.034.

[38] F. Tisseur, K. Meerbergen, The quadratic eigenvalue problem, SIAM review 43 (2) (2001) 235-286.

[39] S. Adhikari, M. I. Friswell, Eigenderivative analysis of asymmetric nonconservative systems, International Journal for Numerical Methods in Engineering 51 (6) (2001) 709-733. doi:10.1002/nme.186.

[40] D. Chronopoulos, Design optimization of composite structures operating in acoustic environments, Journal of Sound and Vibration 355 (2015) 322-344. doi:10.1016/j.jsv.2015.06.028. 
[41] C. A. Felippa, R. W. Clough, The finite element method in solid mechanics, American Mathematical Society, 1970.

[42] T. Coleman, M. A. Branch, A. Grace, Optimization toolbox, For Use with MATLAB. Users Guide for MATLAB 5, Version 2, Release II.

[43] J. S. Jensen, Phononic band gaps and vibrations in one-and twodimensional mass-spring structures, Journal of Sound and Vibration 266 (5) (2003) 1053-1078. doi:10.1016/S0022-460X (02) 01629-2.

[44] P. G. Domadiya, E. Manconi, M. Vanali, L. V. Andersen, A. Ricci, Numerical and experimental investigation of stop-bands in finite and infinite periodic one-dimensional structures, Journal of Vibration and Control 22 (4) (2016) 920-931. doi:10.1177/1077546314537863. 